\title{
Compromiso Organizacional en Trabajadores de Pequeñas y Medianas Empresas
}

\author{
Mtro. Rafael Octavio Félix Verduzco \\ Dra. Claudia García Hernández \\ Mtra. Karla Denisse Espinoza Gracia \\ Dra. Santa Magdalena Mercado Ibarra \\ Dra. Eneida Ochoa Ávila \\ Instituto Tecnológico de Sonora, Departamento de Psicología \\ Email: rafael.felix@itson.edu.mx
}

\begin{abstract}
RESUMEN
El presente trabajo tuvo como objetivo identificar el compromiso organizacional existente en los trabajadores de las Pequeñas y Medianas Empresas para determinar su incidencia en diferentes variables atributivas, así como determinar la confiabilidad general y por dimensión de la escala. El método de la presente investigación es de tipo no experimental transaccional descriptivo, el instrumento utilizado fue el de compromiso organizacional de Meyer y Allen, fue aplicada a 608 empleados que pertenecen a diferentes ámbitos de servicio de las PyMES de Cd. Obregón, Sonora. Después de los análisis correspondientes los resultados muestran un Alpha de .898 para la escala total. Respecto a las pruebas de hipótesis se encontró que los empleados de contrato base tienen un puntaje más alto de compromiso en comparación con los de temporal de igual manera los que ocupan puestos directivos. El constructo de compromiso organizacional se correlaciono con sus diferentes factores como son el normativo, de continuidad y el afectivo, así como con las variables atributivas edad, número de hijos y años de trabajo lo que indica que a mayor sea cada una de estas variables mayor es el nivel de compromiso de los trabajadores.
\end{abstract}

Palabras clave: Compromiso Organizacional, PyMES, comparación de medias.

\section{INTRODUCCIÓN}

Hoy en día la competencia laboral es mucha, sobre todo porque las empresas son muy cuidadosas con el tipo de empleados que seleccionan, esta es la razón por la cual al momento de contratar a alguien e ingresarlo a su compañía se lleva a cabo un procedimiento cuidadoso y detallado, en donde se realizan diferentes estrategias para obtener información sobre el candidato, porque cuanto más minucioso sea el proceso más datos se tendrá sobre la persona y así se sabrá si tiene las competencias necesarias para llegar a ser un trabajador comprometido con su puesto de trabajo, de acuerdo con 
esto, el Compromiso Organizacional (CO), es visto como un factor determinante que influye en la persona para permanecer en el puesto en el que labora o no (Betanzos, Andrade y Paz, 2006).

Lo descrito en el párrafo anterior deja ver la importancia del constructo de compromiso organizacional, ya que, al realizar trabajo sobre este, se facilita la identificación y abordaje de problemáticas relacionadas con la falta de compromiso de los trabajadores por medio de programas de prevención o intervención. Contar con investigaciones al respecto de este constructo fomenta la generación de información sobre la dinámica de esta variable en los trabajadores de una organización.

Al respecto Ruiz (2013), menciona que el compromiso organizacional, ha sido objeto de extenso estudio debido a la importancia que tiene para las empresas, ya que entre más alto sea el compromiso que tenga el empleado con la organización mayor beneficio obtendrá, dado que al contar con personal comprometido éste trabaja para alcanzar los objetivos de la empresa y su superación personal, esto conlleva que la organización alcance el éxito deseado.

En relación a investigaciones de compromiso organizacional a continuación se mencionan algunas de ellas donde se deja ver la importancia del constructo dentro del entorno laboral, es así como Álvarez (2008), realizó un estudio en el que comparó a los empleados de tiempo parcial y de tiempo completo, esto para conocer el tipo de compromiso que presentaba cada uno de ellos en función de la variable del tipo de contrato que tienen en la organización, mediante el análisis que se llevó a cabo se encontró que, el tener un trabajo de tiempo parcial o tiempo completo no influye en el tipo de compromiso que los empleados presentan en su trabajo. Los resultados del estudio que realizó Álvarez mencionan que la empresa, puede mejorar el compromiso de los trabajadores mediante distintas acciones encaminadas al desarrollo del mismo.

Otra de las investigaciones sobre compromiso es la realizada por Omar y Florencia (2008), quienes trabajaron en las relaciones entre los valores individuales y el compromiso organizacional de varias empresas en una muestra de 429 empleados de Argentina. Se aplicó la escala de individualismo-colectivismo y el cuestionario de compromiso organizacional, se les solicitaron datos personales como edad, género, escolaridad, cargo y antigüedad laboral en donde la muestra se clasificó en ocho subgrupos. En los resultados se mostró que los colectivistas desarrollan mayor nivel de compromiso afectivo, también las mujeres y los empleados que tenían mayor antigüedad en la empresa presentaron altos niveles de compromiso afectivo y por otra parte quienes ocupan cargos gerenciales $\mathrm{y}$ empleados de grandes empresas tienen compromiso normativo.

Domínguez, Ramírez y García (2013), trabajaron un estudio de tipo empírico transversal, cuyo objetivo fue analizar el compromiso y clima organizacional que los trabajadores presentaban con la organización, así mismo, la relación que existe entre estos dos constructos, los autores compararon los resultados de estas dos variables con el género, en donde 
obtuvieron niveles similares de compromiso y clima en hombres y mujeres. De igual manera, al segmentar las muestras por edad los trabajadores con más de 40 años de edad, aumentan el compromiso con la organización en las dimensiones afectivas y normativas con un nivel alto.

Por otro lado Bohrt, Solares y Romero (2014), realizaron un estudio donde se enfocaron en establecer las relaciones entre el Compromiso Organizacional y el Contrato Psicológico con datos de 85 empleados de dos Compañías de Seguros de la ciudad de La Paz en Bolivia, introduciendo variables como edad $y$ antigüedad de los participantes, obteniendo como resultados que a mayor edad se encontraba un mayor grado de compromiso, sobre todo en el componente de continuación, respecto a la antigüedad si ésta se incrementaba, la solidez inicial del Contrato Psicológico (ambos tipos: relacional y transaccional) se deterioraba.

Como se puede observar en los estudios presentados el compromiso es una variable importante a trabajar, ya que al hacerlo ésta fomenta diversos beneficios en la empresa como bien lo indican Betanzos y Paz (2007), quienes exponen que lo primordial del compromiso organizacional es que causa un impacto directo en actitudes y conductas que tenga el trabajador, como pueden ser la aceptación de metas, los valores, la cultura de la organización, así como reducir el ausentismo y la baja rotación del personal, entre otros. Debido a esto, es una variable con relevancia en el ámbito de la psicología organizacional, ya que estas se centran en estudiar los vínculos que se generan entre los empleados y la organización. Así mismo Arciniega (2002), sostiene que existen evidencias de que las organizaciones cuyos integrantes poseen altos niveles de compromiso, son aquellas que registran altos niveles de desempeño y productividad y bajo índices de ausentismo.

De acuerdo a lo mencionado en los párrafos anteriores en el presente trabajo se buscó identificar el compromiso organizacional existente en trabajadores de PyMES de Ciudad Obregón, con motivo de determinar la incidencia de éste en diferentes variables atributivas. Así mismo, se buscó determinar la confiabilidad de la Escala de Compromiso Organizacional dentro del contexto de aplicación del presente trabajo.

\section{MÉTODO}

\section{Participantes}

El tipo de muestreo utilizado fue no probabilístico, esto significa que todos los individuos tienen la misma probabilidad de ser elegidos para formar parte de una muestra, este tipo de método nos asegura la representatividad de la muestra extraída, los sujetos seleccionados fueron de las
Pequeñas y Medianas Empresas (PyMES) de Ciudad Obregón, que pertenecen tanto al giro comercial como de servicio con un total de 608 empleados siendo 282 mujeres y 326 hombres.

\section{Instrumento}

Para determinar el nivel de compromiso organizacional presente y la 
tendencia hacia un determinado componente del compromiso en los empleados, se utilizó la escala de Compromiso Organizacional de Meyer y Allen de 1997. Esta escala comprende seis ítems por cada uno de los tres componentes (Afectivo, Continuidad y Normativo) dando un total de 18 ítems en un formato tipo Likert de cinco puntos, la cual va desde totalmente de acuerdo a totalmente en desacuerdo. Los valores de consistencia (alpha de Cronbach) obtenidos para esta escala en lengua hispana son los siguientes: 0.81 para la escala de compromiso afectivo, 0.48 para la escala de compromiso de continuidad y 0.82 para la escala de compromiso normativo (Arias, 1998).

\section{Procedimiento}

Para el presente trabajo se realizó el levantamiento de información en diferentes PyMES de Ciudad Obregón, donde se solicitó permiso previo, contactando con los dueños o encargados de las mismas para aplicar el instrumento que permitan evaluar el compromiso organizacional. El levantamiento se realizó por medio de diversos encuestadores, los cuales se asignaron a las diferentes empresas, de tipo comercial y de servicio, una vez levantada la información se procedió a foliar, capturar en SPSS y limpiar la base de datos para asegurar una captura objetiva, posteriormente se realizaron análisis descriptivos para obtener los primeros resultados y se corrieron comparaciones de medias por medio de $\mathrm{T}$ de Student para conocer la existencia de diferencias significativas entre los resultados del instrumento y algunas variables atributivas, posteriormente se realizó una correlación con motivo de conocer la relación presente entre las variables del instrumento y los datos atributivos.

\section{RESULTADOS}

En el presente apartado, se abordan los resultados obtenidos con base al análisis de la información recabada por medio de los instrumentos aplicados. Primeramente, se muestran los datos de consistencia interna obtenidos para ambos instrumentos con la finalidad de conocer la confiabilidad obtenida, posteriormente se presenta la información derivada de las pruebas de hipótesis para identificar el valor significativo del constructo estudiado en las variables atributivas y finalmente las correlaciones obtenidas con motivo de conocer que variables podrían considerarse más significativas al momento de trabajar este constructo dentro del contexto participante.

\section{Consistencia interna}

Al realizar la prueba alpha de cronbach para ambos instrumentos se obtuvieron los siguientes resultados. $\mathrm{La}$ consistencia interna de la escala total de compromiso tuvo un $\alpha$ de Cronbach de 0.898 y los siguientes valores para cada una de sus subescalas: Compromiso afectivo, 0.824; Compromiso normativo, 0.742 ; Compromiso de continuidad, 0.714. Lo anterior deja ver como de forma general, así como en cada una de sus subescalas la prueba muestra un valor de alpha entre los 
rangos de aceptable y razonable de acuerdo con lo propuesto por George y Mallery (2003), lo cual indica que el instrumento presenta una adecuada consistencia interna.

\section{Comparación de medias}

Se realizó una $\mathrm{T}$ de Student sobre el compromiso organizacional y sexo, encontrando que en general no existen diferencias significativas por sexo entre el total de los participantes (Véase tabla 1).

Tabla 1. Prueba T. Sexo y Compromiso

\begin{tabular}{lllll}
\hline & Hombre & Mujer & $\mathrm{T}$ & $\mathrm{p}$ \\
\hline Compromiso general & 59.05 & 57.01 & 1.917 & .056 \\
Compromiso afectivo & 20.16 & 19.40 & 1.806 & .071 \\
Compromiso normativo & 19.47 & 18.74 & 1.861 & .063 \\
Compromiso de continuidad & 19.42 & 18.87 & 1.457 & .146 \\
\hline
\end{tabular}

Posteriormente se comparó el tipo de contrato con el compromiso general y sus dimensiones, donde de acuerdo a la tabla 2 existen diferencias significativas entre el compromiso y el tipo de contrato, ya que el personal que cuenta con un contrato base con la empresa presenta un puntaje mayor de compromiso general, afectivo, normativo y de continuidad a diferencia del que es por contrato temporal.

Tabla 2. Prueba T. Tipo de contrato y Compromiso

\begin{tabular}{lllll}
\hline & Temporal & Base & T & $\mathrm{p}$ \\
\hline Compromiso general & 55.50 & 58.96 & -2.829 & $\mathbf{. 0 0 5}$ \\
Compromiso afectivo & 18.61 & 20.20 & -3.290 & $\mathbf{. 0 0 1}$ \\
Compromiso normativo & 18.37 & 19.38 & -2.238 & $\mathbf{. 0 2 6}$ \\
Compromiso de continuidad & 18.51 & 19.38 & -1.983 & $\mathbf{. 0 4 8}$ \\
\hline
\end{tabular}

Por otra parte, se realizaron análisis sobre el compromiso y el tipo de puesto de los trabajadores, encontrando diferencias significativas sobre el compromiso general, afectivo y normativo ya que como se puede ver en la tabla 3, los directivos tienen puntajes mayores dentro de estos tipos de compromiso comparado con los otros puestos, mientras que en el factor de compromiso de continuidad no se encontraron diferencias significativas. 
Tabla 3. ANOVA. Tipo de puesto y Compromiso

\begin{tabular}{lllllc}
\hline & Operativo & Administrativo & Directivo & F & P \\
\hline Compromiso general & 57.39 & 60.37 & 66.36 & 4.651 & $\mathbf{. 0 1 0}$ \\
Compromiso afectivo & 19.42 & 21.02 & 24.55 & 9.362 & $\mathbf{. 0 0 0}$ \\
Compromiso normativo & 18.90 & 19.75 & 23.27 & 5.636 & $\mathbf{. 0 0 4}$ \\
Compromiso de continuidad & 19.08 & 19.60 & 18.55 & .676 & .509 \\
\hline
\end{tabular}

En la tabla 4, se aprecia el compromiso general y sus dimensiones con los años laborados en la empresa, donde se encontró diferencias significativas en la comparación de estas variables, observándose puntajes mayores de compromiso general, normativo y de continuidad al tener de dos a tres años laborando en la organización. $\mathrm{Y}$ en el compromiso afectivo al tener tres años en adelante.

Tabla 4. ANOVA. Años laborados y Compromiso

\begin{tabular}{lcccccc}
\hline & $\begin{array}{c}\text { 0 a 1 } \\
\text { año }\end{array}$ & $\begin{array}{l}\text { 1 a 2 } \\
\text { años }\end{array}$ & $\begin{array}{c}\text { 2 a 3 } \\
\text { años }\end{array}$ & $\begin{array}{l}\text { 3 en } \\
\text { adelante }\end{array}$ & F & P \\
& & & & & & \\
\hline Compromiso general & 55.56 & 58.22 & 61.67 & 60.59 & 6.963 & $\mathbf{. 0 0 0}$ \\
Compromiso afectivo & 18.74 & 19.36 & 21.09 & 21.45 & 10.742 & $\mathbf{. 0 0 0}$ \\
Compromiso normativo & 18.55 & 19.14 & 20.25 & 19.58 & 3.042 & $\mathbf{. 0 2 8}$ \\
Compromiso de continuidad & 18.27 & 19.72 & 20.33 & 19.56 & 5.839 & $\mathbf{. 0 0 1}$ \\
\hline
\end{tabular}

Una vez realizado los análisis de comparación de medias se procedió a la realización de correlaciones entre los factores de la escala y las variables atributivas como se indica en la tabla 5, en donde, se puede observar que el compromiso organizacional presenta una alta correlación positiva entre sus factores (afectivo, normativo y de continuidad), también el compromiso organizacional junto con sus factores correlacionan de manera positiva con los años trabajados en la empresa, número de hijos y la edad de los trabajadores, dejando de lado el nivel educativo con el cual no presentan relación, así mismo las horas laboradas no presentan relación con alguna de las variables de compromiso, finalmente la edad se muestra como una variable a considerar ya que correlaciona con todas las variables de este trabajo, tanto para el constructo de compromiso como los atributos de los participantes. 
Tabla 5. Correlación. Compromiso con variables atributivas

\begin{tabular}{|c|c|c|c|c|c|c|c|c|c|}
\hline & $\mathrm{CO}$ & $\mathrm{CA}$ & $\mathrm{CN}$ & $\mathrm{CC}$ & $\mathrm{HL}$ & AT & $\mathrm{NE}$ & $\mathrm{NH}$ & ED \\
\hline $\mathrm{CO}$ & 1 & & & & & & & & \\
\hline CA & $.903^{* *}$ & 1 & & & & & & & \\
\hline $\mathrm{CN}$ & $.919^{* *}$ & $.774^{\star *}$ & 1 & & & & & & \\
\hline $\mathrm{CC}$ & $.858^{* *}$ & $.629^{* *}$ & $.691^{\star * *}$ & 1 & & & & & \\
\hline $\mathrm{HL}$ & .037 & .043 & .027 & .027 & 1 & & & & \\
\hline AT & $.169^{* \star}$ & $.221^{\star \star}$ & $.102^{*}$ & $.126^{\star \star *}$ & $.105^{\star *}$ & 1 & & & \\
\hline $\mathrm{NE}$ & .023 & .057 & .010 & -.009 & -.031 & $.122^{* *}$ & 1 & & \\
\hline $\mathrm{NH}$ & $.113^{* *}$ & $.117^{\star *}$ & $.099^{*}$ & $.086^{*}$ & .009 & $.250^{\star *}$ & $-.249^{* *}$ & 1 & \\
\hline $\mathrm{ED}$ & $.135^{\star \star *}$ & $.149^{* \star}$ & $.120^{\star \star *}$ & $.089^{*}$ & $.103^{*}$ & $.438^{\star \star *}$ & $-.193^{\star *}$ & $.629^{\star *}$ & 1 \\
\hline
\end{tabular}

\section{DISCUSIÓN}

Referente a los resultados obtenidos después de la aplicación del instrumento, se puede concluir que es una escala confiable ya que cuenta con una consistencia interna de .898 total, así mismo, en cuanto a las dimensiones que la conforman se obtuvo un valor de Alpha de .824 para la dimensión de afectivo, .742 para la normativa y .714 para la de continuidad, lo que indica una adecuada consistencia interna entre las áreas que conforman el instrumento. Comparando los resultados con el estudio de Barraza, Acosta y Ledesma (2009), en el cual se utilizó la misma escala de compromiso, pero en el Estado de Durango, la consistencia interna es mayor para este trabajo que para el de los autores anteriores, ya que ellos obtuvieron una Alpha de Cronbach total de 0.81. Por otro lado, Uribe, Moreno y Martínez (2001), obtuvieron coeficientes de $.81, .48$ y .82 (Compromiso afectivo, normativo y de continuidad) resultando menor que en el presente trabajo. Por último, los autores Meyer, Allen y Smith (1993), reportaron en su estudio coeficientes de .82 para afectivo, .74 para continuidad y .83 para normativo, los cuales son ligeramente mayores que en este trabajo.

Para el presente estudio no hay diferencias significativas en relación al sexo de los participantes, al igual que en el estudio de Loli (2006), quien trabajo el compromiso relacionándolo con algunas variables demográficas, encontrando que no 
existen diferencias significativas respecto al compromiso organizacional cuando se compara hombres con mujeres. Lo anterior deja ver que al hablar de compromiso, el sexo no es determinante para su estudio.

Así mismo, para el compromiso y el tipo de contrato se mostró que el personal de base está más comprometido con la empresa en comparación con los de contrato temporal, lo cual significa que los trabajadores tienen un compromiso por permanecer dentro de la empresa, ya sea la causa por sentimientos afectivos hacia la organización o presiones y necesidades monetarias.

En lo referente al puesto de trabajo se encontró, que si existen diferencias significativas con el compromiso organizacional, ya que se mostró que los directivos tienen más alto compromiso comparado con los otros puestos. Por su parte Arias, Varela, Loli y Quintana (2014), en su investigación encontraron que no hubo significancia estadística con la jerarquía del puesto.

Al comparar los años laborados con el compromiso organizacional y sus factores, existe una diferencia significativa, ya que arrojo que a más años laborados en la empresa mayor es el nivel de compromiso, de igual manera para la edad. Dichos resultados concuerdan con lo que señalan Blanco y Castro (2011), quienes encontraron en su estudio que a medida que la antigüedad se incrementa mayor es el nivel de compromiso, así como a mayor edad de los empleados tienen un mayor grado de compromiso.

En lo que se refiere a las correlaciones se encontró que efectivamente el compromiso organizacional tiene una relación positiva con el compromiso afectivo, normativo, y de continuidad, ya que estas suelen relacionarse entre sí al hablar de este constructo. También se identificó que los factores de la escala correlacionaron positivamente con las variables atributivas como años de trabajo, número de hijos y la edad, lo que significa que a cuanto mayor sea cada una de ellas, mayor es el nivel de compromiso. En el estudio de Vargas, Soto y Rosas (2014), se encontró en cuanto a la edad resultados similares al presente trabajo, ya que mencionaban los autores que a mayor edad se manifestaban correlaciones positivas y estadísticamente significativas para los elementos normativo y afectivo, lo que implicaría que a mayor edad aumenta el nivel de compromiso en esos dos elementos. Por otra parte, en el estudio de Bohrt, Solares y Romero (2014), respecto a la antigüedad de los empleados se observó que el compromiso organizacional bajaba después de permanecer un año en la empresa, e incrementaba después de los cinco años, en cuanto a la edad, de igual manera se encontró que el compromiso en sus diferentes componentes se incrementa de manera sostenida con la edad. 


\section{REFERENCIAS}

Álvarez G. 2008. Determinantes del compromiso organizacional. ¿Están los trabajadores a tiempo parcial menos comprometidos que los trabajadores a tiempo completo?, Cuadernos de Estudios Empresariales, volumen \#18, p. 73-88

Arciniega LM. 2002. Compromiso Organizacional en México: ¿Cómo hacer que la gente se ponga la camiseta? Dirección Estratégica, Revista de Negocios del ITAM, 2(1), 21-23.

Arias GF. 1998. Los cuestionarios de compromiso personal y clima organizacional. Ponencia presentada en el IV Foro Nacional de Investigación en las Disciplinas FinancierasAdministrativas, Facultad de Contaduría y Administración de la Universidad Nacional Autónoma de México.

Arias Galicia F, D Varela Sosa, A Pineda, M Quintana. 2014. El compromiso organizacional y su relación con algunos factores demográficos y psicológicos. Revista de Investigación en Psicología, 6(2), 13-25.

doi:http://dx.doi.org/10.15381/rinvp.v6i2.5147

Barraza A, M Acosta y Z Ledesma. (2009. Compromiso organizacional de los docentes de una institución de educación media superior. Recuperado de:

http://www.comie.org.mx/congreso/memoriaelectroni ca/v10/pdf/area_tematica_16/ponencias/0414-F.pdf

Betanzos N, P Andrade, y F Paz. 2006. Compromiso organizacional en una muestra de trabajadores mexicanos. Revista de Psicología del Trabajo y de las Organizaciones, 22 (1). 25-43.

Betanzos N y F Paz. 2007. Análisis psicométrico del compromiso organizacional como variable actitudinal. Anales de psicología, 23 (2). 207-215.

Blanco M \& Ch P Castro. 2011. Análisis comparativo del compromiso organizacional manifestado por madres y mujeres sin hijos. Ajayu. Órgano de Difusión Científica del Departamento de Psicología de la Universidad Católica Boliviana "San Pablo", 9 (2), 215-233.

Böhrt R, L Solares y C Romero. 2014. Evolución del contrato psicológico y el compromiso organizacional con la edad y la antigüedad. Ajayu. Órgano de Difusión Científica del Departamento de Psicología de la Universidad Católica Boliviana "San Pablo", 12 (1), 33-63.

Domínguez Aguirre L, A Ramírez Campos y A García Méndez. 2013. El clima laboral como un elemento del compromiso organizacional. Revista Nacional de Administración, 4(1), 59-70.

Meyer J y N Allen. 1997. Commitment in the workplace: Theory, research and application, California Publications, 69 (3), 372-378.

Meyer J, N Allen y CA Smith. 1993. Commitment to Organizations and Occupations: Extension and Test of a Three-Component Conceptualization. Journal of Applied Psychology, 78, 538-551.

https://doi.org/10.1037/0021-9010.78.4.538

Omar Ay A Urteaga. 2008. Valores personales y compromiso organizacional. Enseñanza e Investigación en Psicología, 13 (2), 353-372.

Loli Pineda AE. 2006. Compromiso organizacional de los trabajadores de una universidad pública de Lima y su relación con algunas variables demográficas, en Revista de Investigación en Psicología, Vol. 9, No. 1, pp.37-67.

Ruiz J. 2013. El compromiso organizacional: un valor personal y empresarial en el marketinginterno. Revista de Estudio Empresariales, 1 (13). 67-86.

Uribe JF, G Moreno y E Martínez. 2001. Compromiso Organizacional y Maquiavelismo en ejecutivos mexicanos. XII Congreso Nacional de Psicología del Trabajo y IX Congreso Iberoamericano de Recursos Humanos

Vargas J, J Soto y K Rosas. 2014. La relación del Compromiso Organizacional con el nivel de estudios, la edad y el género en trabajadores de la industria del calzado en el León, Guanajuato. OIDLES (Observatorio 85 Iberoamericano del Desarrollo Local y la Economía Social), 16, 1. Recuperado de http://www.eumed.net/rev/oidles/16/sociodemografia .pdf 